\title{
Presence of a functional but dispensable Nuclear Export Signal in the HTLV-2 Tax protein Sébastien A Chevalier ${ }^{1}$, Laurent Meertens ${ }^{1}$, Sara Calattini ${ }^{1}$, Antoine Gessain ${ }^{1}$, Lars Kiemer ${ }^{2}$ and Renaud Mahieux*1
}

\author{
Address: ${ }^{1}$ Unité d'Epidémiologie et Physiopathologie des Virus Oncogènes, Institut Pasteur, Paris, France and ${ }^{2}$ Center for Biological Sequence \\ Analysis, BioCentrum-DTU, The Technical University of Denmark, Building 208 DK-2800, Lyngby, Denmark \\ Email: Sébastien A Chevalier - schevali@pasteur.fr; Laurent Meertens - lmeerten@aecom.yu.edu; Sara Calattini - scalatt@pasteur.fr; \\ Antoine Gessain - agessain@pasteur.fr; Lars Kiemer - lars@cbs.dtu.dk; Renaud Mahieux* - rmahieux@pasteur.fr \\ * Corresponding author
}

Published: 14 November 2005

Retrovirology 2005, 2:70 doi:10.1 186/1742-4690-2-70

This article is available from: http://www.retrovirology.com/content/2///70

(C) 2005 Chevalier et al; licensee BioMed Central Ltd.

This is an Open Access article distributed under the terms of the Creative Commons Attribution License (http://creativecommons.org/licenses/by/2.0), which permits unrestricted use, distribution, and reproduction in any medium, provided the original work is properly cited.

\begin{abstract}
Background: Human T-cell leukemia virus type $\mathrm{I}$ and type 2 are related human retroviruses. HTLV-I is the etiological agent of the Adult T-cell Leukemia/Lymphoma and of the Tropical Spastic Paraparesis/HTLV-I Associated Myelopathy, whereas, HTLV-2 infection has not been formally associated with any T-cell malignancy. HTLV-I and 2 genomes encode, respectively, the TaxI and Tax2 proteins whose role is to transactivate the viral promoter. HTLV-I and HTLV-2 Tax sequences display $28 \%$ divergence at the amino acid level. Taxl is a shuttling protein that possesses both a non canonical nuclear import (NLS) and a nuclear export (NES) signal. We have recently demonstrated that $\operatorname{Tax} I$ and $\operatorname{Tax} 2$ display different subcellular localization and that residues 90 100 are critical for this process. We investigate in the present report, whether Tax 2 also possesses a functional NES.
\end{abstract}

Results: We first used a NES prediction method to determine whether the Tax2 protein might contain a NES and the results do suggest the presence of a NES sequence in Tax2. Using Green Fluorescent Protein-NES (GFP-NES) fusion proteins, we demonstrate that the Tax2 sequence encompasses a functional NES (NES2). As shown by microscope imaging, NES2 is able to mediate translocation of GFP from the nucleus, without the context of a full length Tax protein. Furthermore, point mutations or leptomycin B treatment abrogate NES2 function. However, within the context of full length Tax2, similar point mutations in the NES2 leucine rich stretch do not modify Tax2 localization. Finally, we also show that TaxI NES function is dependent upon the positioning of the nuclear export signal "vis-à-vis" GFP.

Conclusion: HTLV-2 Tax NES is functional but dispensable for the protein localization in vitro.

\section{Background}

HTLV-1 and HTLV-2 are closely related retroviruses that infect T-cells in vivo, with a probable preferential tropism for $\mathrm{CD}^{+}{ }^{+}$and $\mathrm{CD} 8^{+}$cells respectively [1]. HTLV-1 is the eti- ological agent of the Adult T-cell Leukemia/Lymphoma (ATLL) and of the Tropical Spastic Paraparesis/HTLV-1 Associated Myelopathy (TSP/HAM), while HTLV-2 infection, even if originally described in a patient suffering of 
atypical hairy T-cell leukemia, has only been linked to infrequent cases of TSP/HAM "like" disease [2-4]. Both HTLV-1 and HTLV-2 genomes encode a viral transactivator (Tax1 and Tax2 respectively). Tax1 has an oncogenic potential and is responsible for cell-transformation in vitro $[5,6]$. Tax 1 and Tax2 display approximately $75 \%$ nucleotide sequence homology. Strikingly however, several reports have now demonstrated that although the critical functional regions of the proteins are well conserved (i.e. NF- $\kappa \mathrm{B}$ and CREB/ATF activation domains), the two transactivators exhibit a number of major phenotypical differences [1,7-18]. Nevertheless, Tax2 is capable of immortalizing human lymphocytes and, although to a lesser extent than Tax1, of transforming rat cells in vitro $[10,19]$.

Eukaryotic cells are compartmentalized into the cytoplasm and the nucleus by the nuclear envelope [20,21]. The nuclear envelope contains nuclear pore complexes (NPCs), which mediate the traffic of molecules between the two compartments. The nucleo-cytoplasmic traffic of large molecules is regulated by specific nuclear import and export systems. Proteins that contain classical Nuclear Localization Signals (NLSs) are imported into the nucleus by importin $\alpha / \beta$ protein heterodimers. So far, six importin $\alpha$ family members and one importin $\beta$ have been described [22]. Importin $\alpha$ binds to NLS containing proteins, whereupon importin $\beta$ is responsible for the docking of the importin cargo complex to the cytoplasmic side of the NPC, followed by translocation of the complex through the NPC. A classical monopartite NLS consists of a stretch of basic amino acids such as arginines and lysines. Contrary to this, the Nuclear Export Signal (NES) generally consists of a leucine/isoleucine-rich sequence [23]. The classical NES pattern is $\mathrm{L}-\mathrm{x}(2,3)$ - [LIVFM]$\mathrm{x}(2,3)$-L-X- $[\mathrm{LI}]$, where $\mathrm{L}$ can either be $\mathrm{L}, \mathrm{I}, \mathrm{V}, \mathrm{F}$ or $\mathrm{M}$, but many known NES regions do not conform to these limitations [24]. For example, the spacing between the hydrophobic residues is variable and NES regions can also be rich in glutamate, aspartate and serine [23]. The first nuclear export pathway to be discovered involved the chromosome region maintenance 1 (CRM1) receptor, exporting proteins containing a nuclear export signal (NES) [25]. CRM1 binds to a Nuclear Export Signal (NES)-containing protein and to the NPC. Several ways of regulating NES-dependent export have been reported, including masking or unmasking the NES and post-translational modifications of the NES-containing protein [26].

Cellular fractionation and immunofluorescence experiments performed with HTLV-1 infected and Tax1 transfected cells have demonstrated that Tax 1 was present both in the nuclear and cytoplasmic fractions. However, the distribution of the protein between these two compart- ments is unequal and depends on the cell-line used [2731]. The Tax1 48 amino terminal sequence contains a non-canonical functional NLS [32] that allows the protein to enter the nucleus, where Tax1 localizes to discrete nuclear bodies (also called Tax Speckled Structures (TSS) [33]. In addition, Tax1 also contains a "Rev-like" Nuclear Export Signal (NES) spanning from amino acid 189 to 202. This NES is insensitive to leptomycin B within the context of the full-length protein [27]. Both localization signals (NLS and NES) are likely to be involved in the shuttling of Tax1, but this process is still not clearly understood [34].

We have recently reported that, although Tax2 contains a functional NLS domain, the protein localizes predominantly to the cytoplasm in HTLV-2 immortalized or transformed infected T-cells as well as in Tax 2 transfected cells [16]. These results were further confirmed in another laboratory [35] which also demonstrated that the NLS domain was confined to the 40 first $\mathrm{N}$-terminal amino acids. We also demonstrated that the region spanning amino acids 90 to 100 was critical for Tax 2 localization [16]. The recent report of a Tax1 NES sequence prompted us to examine the possible presence of a NES in Tax2. In addition to the 90-100 domain, this sequence could serve as a second domain involved in Tax2 localization. We show in this report that, although HTLV-2 Tax protein contains a NES sequence that is active without the context of a full-length protein, this domain is dispensable for the Tax2 localization.

\section{Results \\ HTLV-2 Tax protein sequence contains a putative NES domain}

We lately demonstrated that the HTLV-2 Tax protein has an intracellular localization that is different from that of Tax1, both in infected and transfected cells (i.e. Tax2 localizes more to the cytoplasm than Tax1) and that, within the Tax sequence, the 90-100 domain was critical for the protein localization [16]. These results were confirmed lately [35]. Another recent article reported that, in addition to the previously characterized NLS, HTLV-1 Tax protein also contains a Nuclear Export Signal (NES) comprising amino acids 189 to 202 (KRIEELLYKISLTT). This sequence contains a string of hydrophobic amino acids (I191, L195, I198 and L200) [27] and has the ability to redirect the Green Fluorescent Protein (GFP) to the cytoplasm. Within the Tax1 NES sequence, residues L195 (formerly named L194 [27]) and L200 appear to be critical for the Tax1 NES function. As an example, when Tax1 L200 is mutated to an alanine, the GFP-Tax1 localization is altered [27].

In order to identify whether Tax2 contains a similar sequence, we first used the NetNES prediction method 
Tax2 sequence

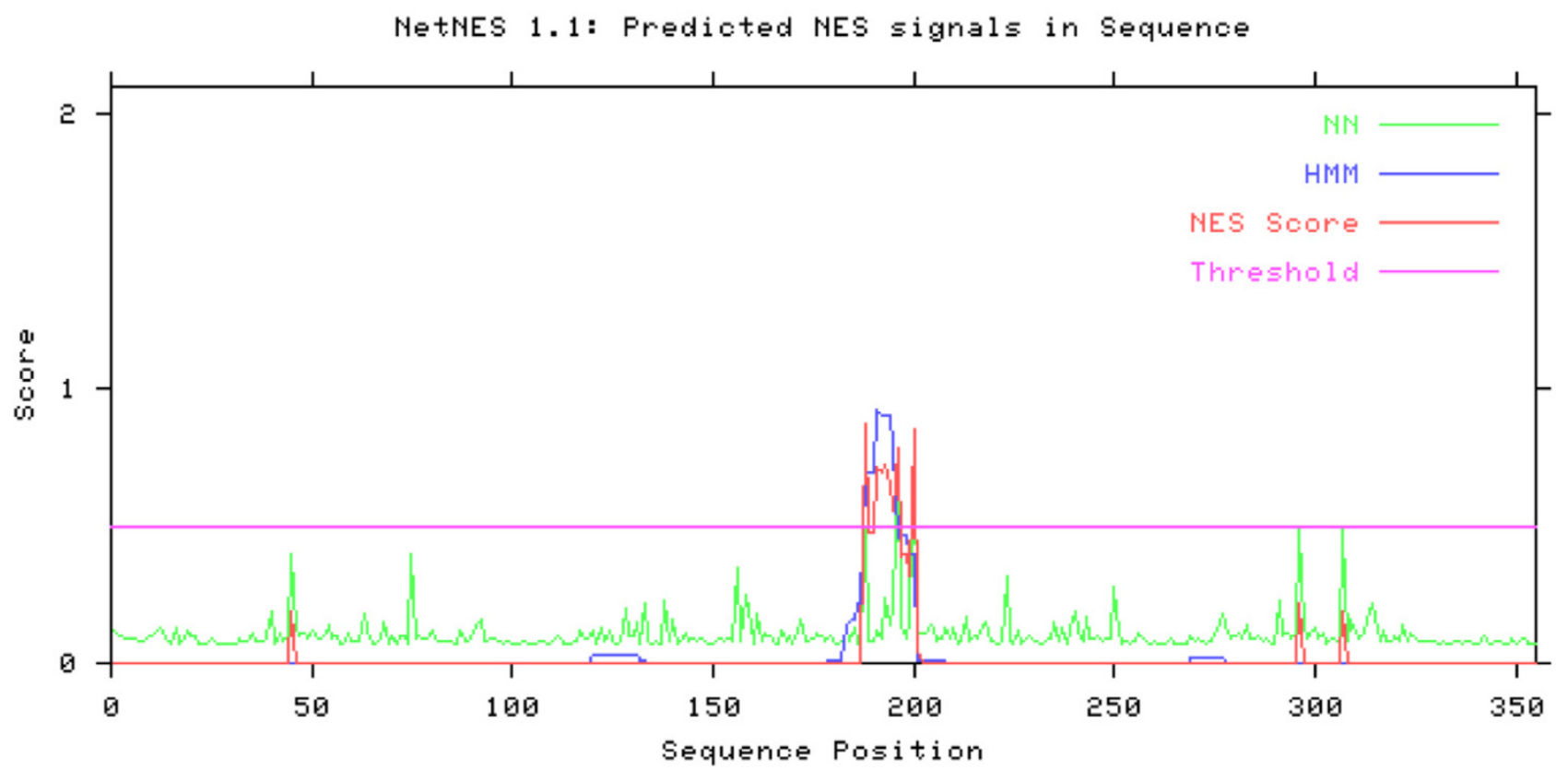

\begin{tabular}{|c|c|c|c|c|}
\hline \#Seq-Pos-Residue & ANN & HMM & NES & Predicted \\
\hline Sequence-188-I & 0499 & 0.680 & 0876 & Yos \\
\hline Sequence-189-K & 0.079 & 0.693 & 0.480 & - \\
\hline Sequence-190-R & 0.077 & 0.693 & 0.480 & - \\
\hline Sequence-191-L & 0.122 & 0.926 & 0.712 & Yes \\
\hline Sequence-192-E & 0.087 & 0.900 & 0.689 & Yes \\
\hline Sequence-193-E & 0.242 & 0.900 & 0.721 & Yes \\
\hline Sequence-194-L & 0.143 & 0.900 & 0.650 & Yes \\
\hline Sequence-195-I & 0.212 & 0.779 & 0.555 & Yes \\
\hline Sequence-196-Y & 0.744 & 0.459 & 0.784 & Yes \\
\hline Sequence-197-K & 0.143 & 0.459 & 0.388 & - \\
\hline Sequence-198-M & 0.093 & 0.467 & 0.397 & - \\
\hline Sequence-199-F & 0.170 & 0.398 & 0.314 & - \\
\hline Sequence-200-I & 0.746 & 0.398 & 0.850 & Yes \\
\hline
\end{tabular}

\section{Figure I}

Output after submission of the complete Tax2 amino acid sequence to the NetNES website [23]. Output consists of two parts; one is a listing of all residues with the individual scores noted (see column heading), the other is a graphical plot of the values given in the table. The prediction server calculates the NES score from the HMM and Artificial Neural Network (ANN) scores but all three values are given for each residue. If the calculated 'NES score' exceeds the threshold, then that particular residue is expected to participate in a nuclear export signal. This is denoted with a 'Yes' in the column "Predicted". 
[23]. (Figure 1). From this analysis, residue L188 of Tax2 was predicted to be part of the Tax 2 NES domain. Interestingly, L188 is absent from the sequence of Tax 1, where the position is occupied by a tyrosine (data not shown). The amino acid comparison of Tax 1 and Tax 2 also reveals that the two sequences are very similar in the 189 to 202 amino acids region, with observed differences at positions 191, 198, 199 and 201 (Figure 2A).

We set out to investigate whether, despite these differences, the Tax 2 putative NES was functional. To this end, we affixed the 189-202 amino-acid domain of Tax 2 to the $\mathrm{N}$-terminus of the GFP sequence (NES2-EGFP) using the pEGFP-N1 vector as previously reported [27]. The NES2EGFP construct was then transiently transfected in $293 \mathrm{~T}$ (data not shown) and Hela cells, as these cells have frequently been used for Tax localization studies $[17,27]$. As a positive control, the NES Tax1 sequence was also fused to the N-terminus of the GFP (NES1-EGFP). In the absence of a Tax NES sequence, the GFP protein is nearly equally distributed between the cytoplasm and the nucleus of the transfected cells ([16] and data not shown). However, the GFP signal was almost entirely cytoplasmic when the protein was fused to the Tax 2 putative NES (Figure $2 \mathrm{~B}$ panel a and Figure $2 \mathrm{C}$ for fractionation). This suggests that this latter sequence mediates an active transport of GFP in vitro. Unexpectedly, and contrary to a previous report [27], the NES1-EGFP fusion protein was diffused in both the nucleus and the cytoplasm with a nuclear content that was much higher than that of NES2-EGFP (Figure 2B panels a vs. $\mathrm{b}$ and Figure 2C). We obtained and sequenced the construct that has been used in Dr Wigdahl's laboratory and the sequence results showed that the Tax1 NES domain had been cloned to the C-terminus part of the GFP rather than to the N-terminus (data not shown). Consequently, a second series of recombinant plasmids was made using the pGFP-C3 vector, allowing for a GFP C-terminal fusion construct. As with the NES2EGFP construct, GFP-NES2 was mostly cytoplasmic (Figure 2B panel $\mathrm{C}$ ), while, under these experimental conditions, GFP-NES1 was also, as previously published, preponderant in the cytoplasm (Figure 2B panel d). Interestingly, subcellular fractionation experiments clearly demonstrated that, even in that case, the GFP-NES1 nuclear fraction was more abundant than that of GFPNES2 (Figure 2C right panel). Altogether, these results suggest that, without the context of a full length protein, Tax2 NES domain is active both when fused to the N- or to the C-terminus part of the GFP, while Tax1 NES functions more efficiently when fused to the C-end of GFP.

\section{Within Tax2 NES sequence, several leucine residues are critical for a CRM-I dependent function}

We next investigated whether Tax2 NES activity was dependent upon the CRM-1 pathway. To this end, Hela cells were transfected with the different NES constructs (Figure 3A), with or without leptomycin B (LMB). LMB blocks CRM1-dependent nuclear export and has been used extensively to probe this process [36]. In the presence of LMB, GFP-NES2 localizes to the nucleus, suggesting that the CRM-1 pathway is involved in the shuttling of the fusion protein (Figure 3B panel b). As a control, incubation of the GFP-NES transfected cells with methanol (the solvent which has been used to dissolve LMB in panel b), had no effect (Figure 3B panel a). Leucine 195 (formerly named 194 [27]) has been shown to be critical for the NES1 ability to export the GFP protein via the CRM-1 dependant pathway. Since the sequence of NES2 also contains a leucine at position 195, we mutated this residue to an alanine. This mutation abrogated the nuclear export of GFP-NES2 (Figure 3B, panel c). As expected, adding LMB to the GFP-NES2 $\mathrm{L}_{195} \mathrm{~A}$ had no effect on the protein localization (Figure 3B, panel d). Mutating leucine 200 to an alanine also suppressed NES function (Figure 3D), while the $\mathrm{L}_{194} \mathrm{~A}$ mutation had no effect (data not shown). Altogether these results confirm that, within the Tax2 NES domain, more than one leucine residues are needed for the function of the export signal. Western blot controls show that the overall protein expression was comparable between the different constructs (Figures 3C and 3E).

\section{Evaluating the role of Tax2 leucine 188}

The NES prediction software results suggested that L188 might be part of NES2 (Figure 1). To evaluate the role of this amino acid in Tax2 NES function we constructed another series of NES-EGFP plasmids, in which amino acid leucine \# 188 was added to the autologous (i.e. NES2) or to the heterologous NES (i.e. NES1) sequences. The constructs were transfected into Hela cells and the expression of the fusion proteins determined by western-blot (Figure 4A). As described above, the NES1-EGFP has a stronger nuclear localization than NES2-EGFP (Figure 4B panels a vs. c). This is correlated with the fractionation experiment (Figure 4C left panel). Remarkably, adding a leucine to the NES1-EGFP sequence improved the "NES" phenotype, since the nuclear fraction is lower in the presence of leucine 188 (Figure 4B panel a vs. $\mathrm{b}$ and Figure 4C right panel). Adding leucine 188 to the ${ }_{189} \mathrm{NES} 2_{202}$-EGFP sequence increased only modestly the percentage of cells in which the signal was cytoplasmic (Figure 4B, panels C vs. d). Altogether, these results demonstrate that a leucine residue at position 188 allows a better export of the NES1EGFP fusion protein. However, this leucine is dispensable in the context of a GFP-NES1 protein.

\section{The localization of GFP-Tax2 is not altered by mutations in the NES}

Although the results presented above have shown that the Tax2 NES represents an active domain in the context of the NES2-EGFP and GFP-NES2 chimera proteins, it was 
A)

189

202

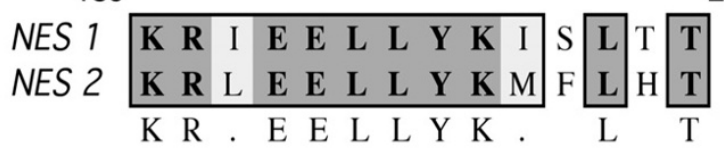

Consensus NES:

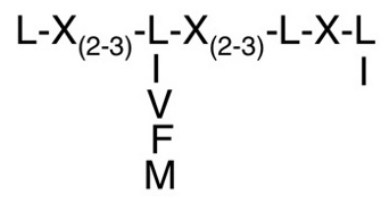

B)

DAPI

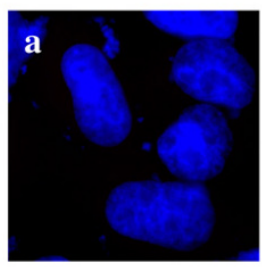

b

NES 1-EGFP

GFP-NES 2

GFP-NES 1
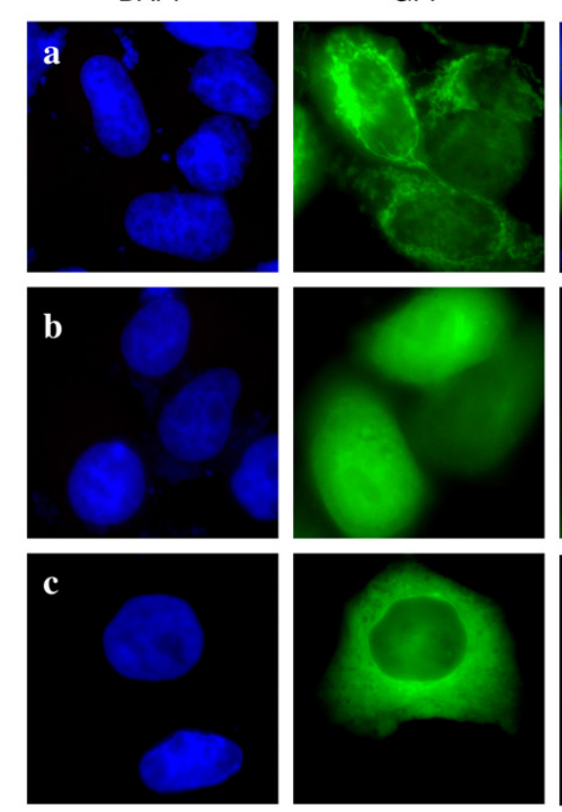

\section{d \\ d}
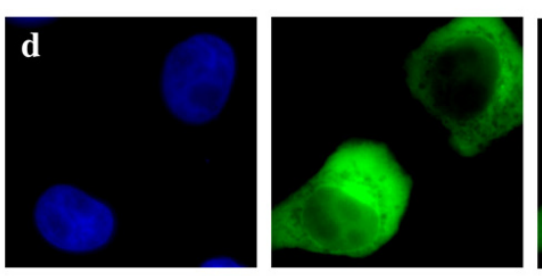

C)

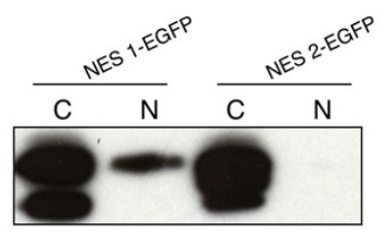

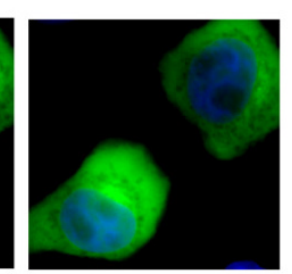

MERGE
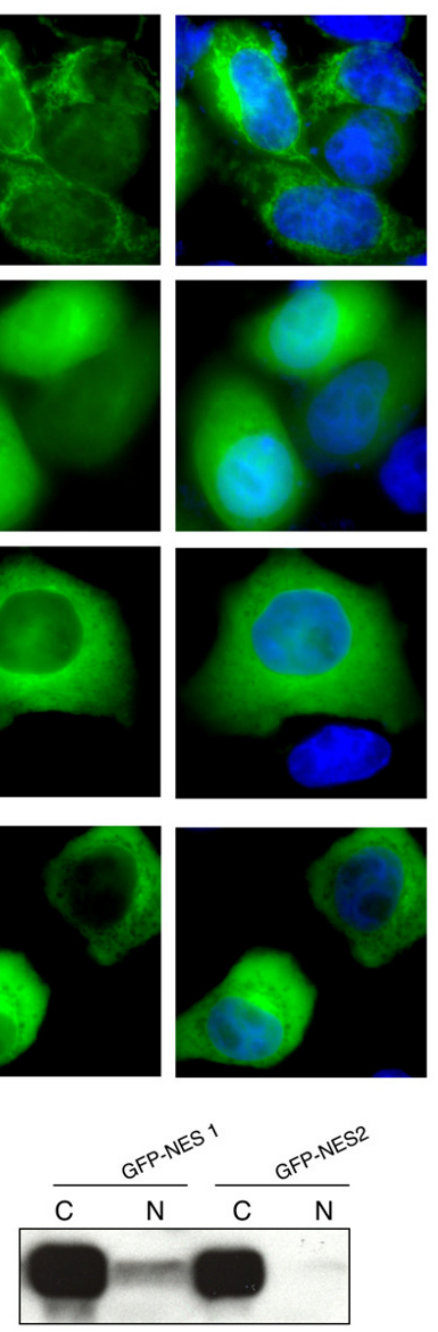

\section{Figure 2}

Without the context of the full length protein, NES2 can redirect GFP to the cytoplasm, while NESI function depends on its positioning vis-à-vis GFP. (A): Sequence alignment of a consensus NES sequence with TaxI NES and Tax2 putative NES. (B): HeLa cells were transiently transfected with NES-EGFP and GFP-NES plasmids. Twenty-four hours after transfection, the cells were washed with PBS, fixed with $4 \%$ paraformaldehyde, mounted with DAPI-containing mounting medium and visualized with a Zeiss Axioplan 2 imaging microscope X40 using a Zeiss Axiocam HRc (color) camera and the Zeiss Apotome software. Images of cells that are representative of the entire population are shown. (C): Western-blot analysis of cytoplasmic and nuclear cell fractions. 293T cell fractions were subjected to electrophoresis on a $10 \%$ TG gel and probed with a GFP antibody. The western-blot results are representative of four independent experiments. 
important to examine the function of the NES2 within the context of the full-length Tax 2 protein. To do this, point mutations were made in the GFP-Tax2 full-length construct, with one or several leucine residues (up to three) mutated within the NES2. We also mutated residue 188 in order to evaluate the role of this amino-acid in the context of the complete protein. Unexpectedly, all these mutated Tax proteins, i.e. GFP-Tax2 $\mathrm{L}_{188} \mathrm{Y}$, GFP-Tax2 $\mathrm{L}_{188} \mathrm{Y}, \mathrm{L}_{191} \mathrm{~A}$, GFP-Tax2 $\mathrm{L}_{188} \mathrm{Y}, \mathrm{LL}_{194-195} \mathrm{AA}, \mathrm{GFP}-\mathrm{Tax} 2 \mathrm{~L}_{200} \mathrm{~A}$, had a predominant cytoplasmic localization and behaved mostly like Tax2 wild-type, i.e. with a strong cytoplasmic localization (Figure 5A, panels c, d, e, f as compared to b), but not like Tax1 (Figure 5A, panel a). Western-blot analysis demonstrated a comparable level of protein expression (Figure $5 \mathrm{~B})$. These results suggest that the presence of a wild-type NES2 is dispensable for exiting the cell nucleus in the context of the full-length Tax2 protein. As a control, we also used a GFP-Tax $1 \mathrm{~L}_{200} \mathrm{~A}$ vector. Strikingly however, in our hands this protein had a localization that was very similar to that of wild-type Tax-1 i.e. strong nuclear signal (data not shown). Indeed, we did not observe a strong localization to the nuclear membrane as it has been previously described. However, we should point out that we have used a GFP-Tax1 construct, while Alefantis et al used a Tax1-EGFP [27]. We cannot rule out the fact that the positioning of Tax $1 \mathrm{~L}_{200} \mathrm{~A}$ vis-à-vis GFP plays a role in the protein localization, although this is unlikely, since we previously observed that the localization of GFP-Tax 1 was similar to that of Tax1-GFP [16].

\section{Discussion}

Both in infected and in transfected cells, Tax 1 and Tax 2 are found in the nucleus and in the cytoplasm in different proportions: Tax 1 being more abundant in the nucleus, while Tax2 is more prone to be found in the cytoplasm $[16,35]$. In the nucleus, Tax 1 and Tax 2 interact with transcription factors and activate the cyclic-AMP response element and activating transcription factor (ATF) binding (CREB/ATF) pathway, while in the cytoplasm the viral transactivators interact with several members of the NF- $\mathrm{KB}$ transduction pathway [5,37]. Tax1/Tax2 activation of CREB/ATF is needed for an efficient viral gene expression, while the permanent activation of NF- $\mathrm{KB}$ has been suggested to be critical, at least in HTLV-1 infected cells, for evading apoptosis. In order to activate the CREB/ATF and NF-kB pathways, both Tax 1 and Tax 2 must therefore shuttle between these two compartments [34].

A Nuclear Export Signal (amino acid 189 to 202) has recently been described in Tax1 [27]. Amino acids 1 to 58 constitute non canonical Nuclear Localization Signals $[16,32]$ in both Tax 1 and Tax2, but amino acids 90 to 100 are also critical for the localization of the viral transactivators [16]. Using prediction software as well as in vitro assays, we now describe another domain of Tax2. This sequence represents a Nuclear Export Signal (NES), with different functional characteristics from that of NES1. For example, the percentage of the GFP-NES2 protein that is present in the nucleus of the transfected cells is slightly different from that of GFP-NES1 protein. In addition, Tax2 NES is functional, no matter if it is fused to the N-terminal or the C-terminal of GFP, which is not the case of NES1 which is more active when fused to the C-terminus of GFP. We have also determined here that the NES of Tax2 can direct nuclear export via the CRM1 pathway, and that point mutations at positions 195 and 200 abrogate NES mediated translocation. All in all, these results demonstrate that the NES sequences of Tax 1 and Tax 2 have different functional profiles reflecting their slightly different sequences, and that the divergent amino acids are likely to be critical for the NES activity. The predictor software suggested that, in the Tax 2 sequence, leucine 188 might also be part of the NES domain. This leucine is absent from Tax 1 and, strikingly, when added to the NES1-EGFP construct, it restores the function of the Tax 1 NES.

However, the most important result of this study is that, within the context of the whole Tax2 protein, mutating one or several leucine residues has no or an extremely limited impact on Tax 2 localization. This could have been indicative of a secondary NES in the sequence being able to mediate translocation on its own, but this theory is not supported by the NetNES computational analysis. Therefore, this hypothesis is very unlikely. It would also disagree with our report that LMB treatment of Tax2 transfected cells did not abolish protein translocation [16]. Hence, we consider that the very modest increase in the GFP-Tax2 nuclear signal observed with some GFPTax2 mutants constructs as compared to GFP-Tax2 is not consistent with a strong use of this NES sequence by Tax 2 . Altogether, these results imply that the Tax 2 protein uses other means of export from the cell nucleus leading to the observed strong cytoplasmic signal. This is consistent with our previous results showing that the 90-100 Tax domain, which does not behave as a NES, is critical for the protein localization [16]. Our results are therefore paradoxical: while Tax1 possesses a NES domain, it localizes predominantly in the nucleus at the equilibrium, whereas Tax-2, whose NES sequence is dispensable, has a predominant cytoplasmic localization.

In conclusion, without the context of the protein, both Tax 1 and Tax 2 seem to possess working nuclear export signals. If one regards the nuclear localization signal and nuclear export signal as competing forces, the Tax2 NES seems to be a more efficient mediator than that of Tax 1 in terms of cytoplasmic versus nuclear abundance of the proteins without the context of a full-length protein. This observation is supported by the computational analysis as 
A)

\begin{tabular}{|c|c|}
\hline & 202 \\
\hline NES 2 & 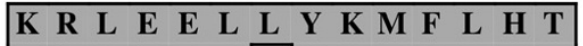 \\
\hline NES 2 L195A & 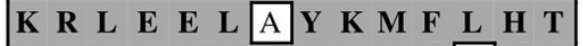 \\
\hline L200A & 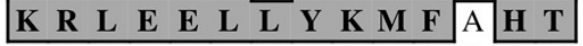 \\
\hline
\end{tabular}

B)
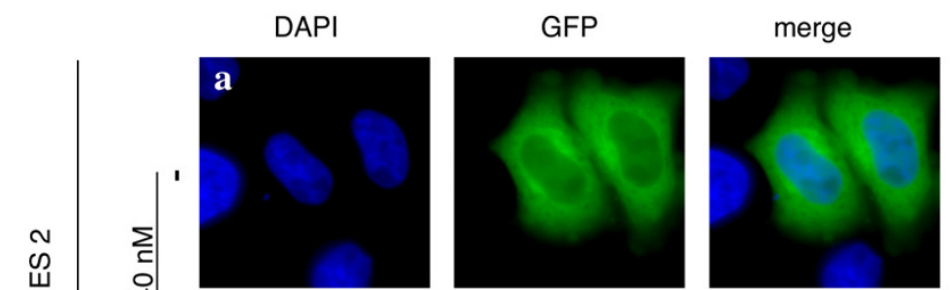

荘
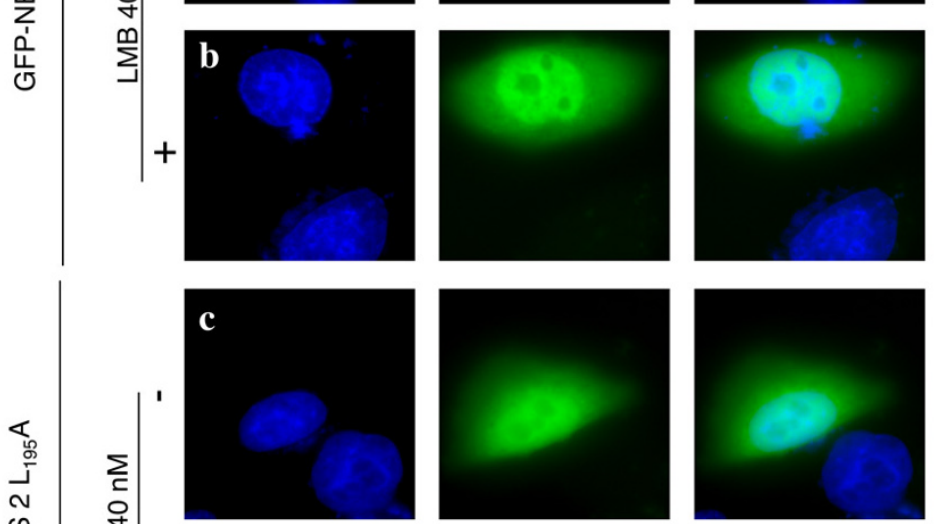

C)
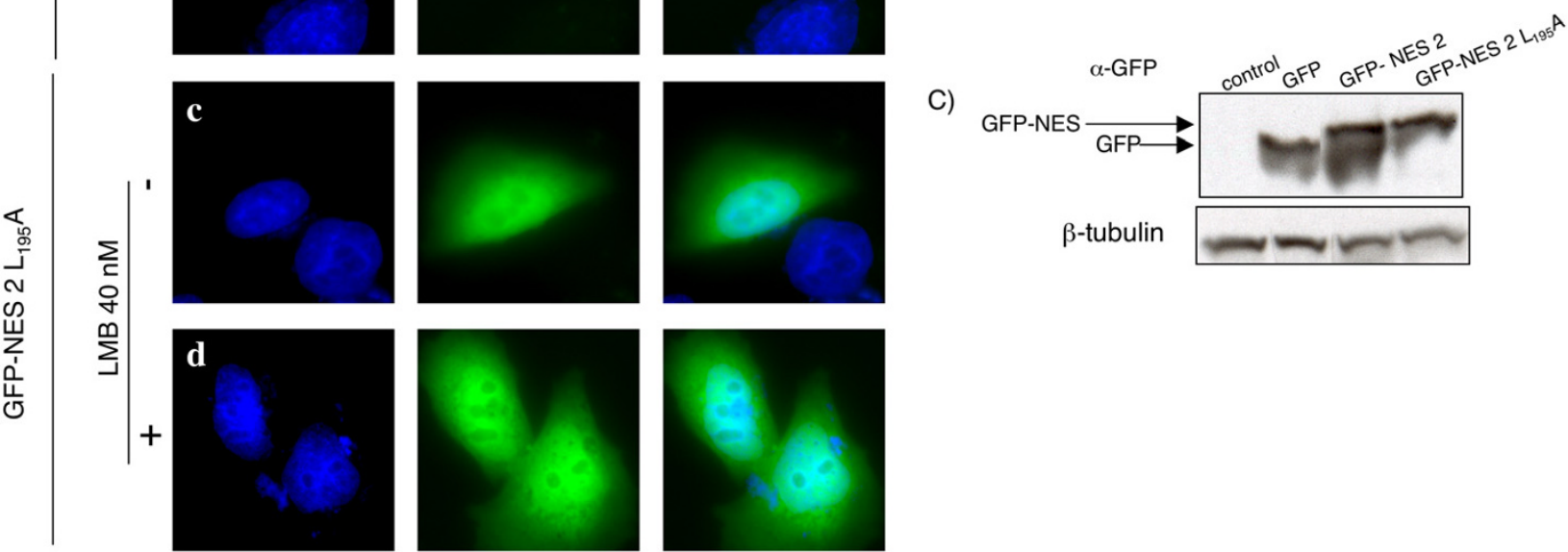

D)

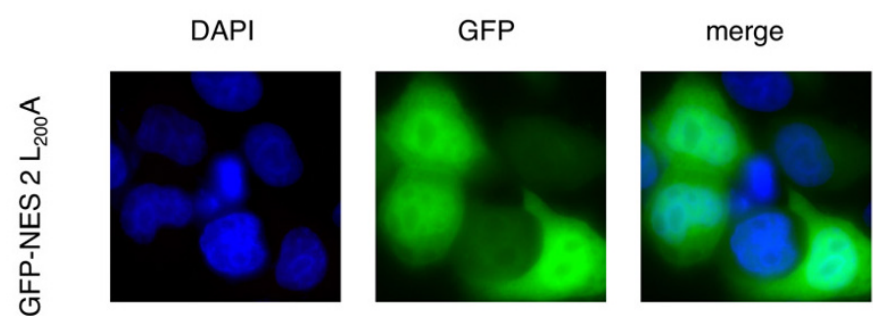

E)

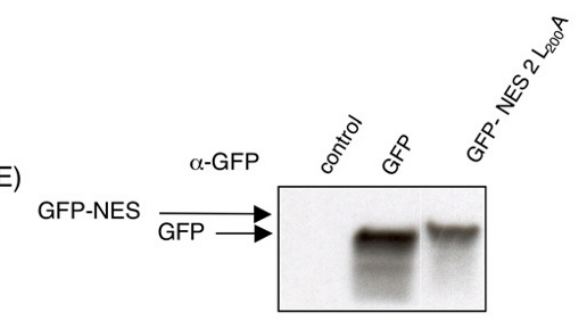

$\beta$-tubulin

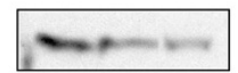

Figure 3

Nucleocytoplasmic distribution of GFP-NES2 is altered after incubation with leptomycin B or by single point mutations. (A): Sequence alignment of wild-type Tax2 and Tax2 GFP-NES mutants. (B and D): HeLa cells were transiently transfected with the different GFP-NES plasmids. Eighteen hours post transfection, transfected cells were treated with leptomycin B (40 nM) or methanol for 3 hours. Cells were then washed, fixed, mounted with DAPI-containing medium and visualized with a Zeiss Axioplan 2 imaging microscope X40 using a Zeiss Axiocam HRc (color) camera and the Zeiss Apotome software. Images of cells that are representative of the entire population are shown. ( $\mathrm{C}$ and $\mathrm{E}$ ): Western-blot analysis of GFP and GFP-NES proteins. $293 \mathrm{~T}$ cell lysates $(70 \mu \mathrm{g})$ were subjected to electrophoresis on a $10 \%$ TG gel and probed with GFP or $\beta$-tubulin antibodies. 
A)

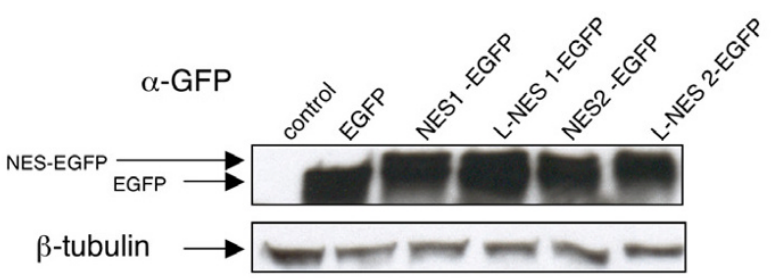

B)

\section{DAPI}
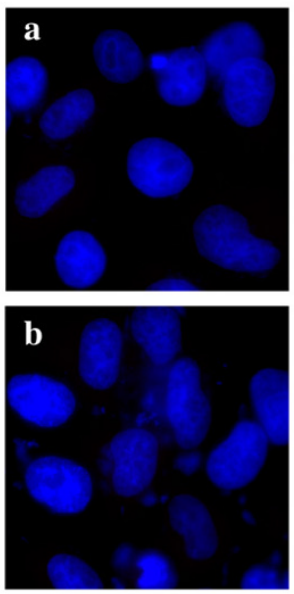

L- NES 1-EGFP

NES 2-EGFP
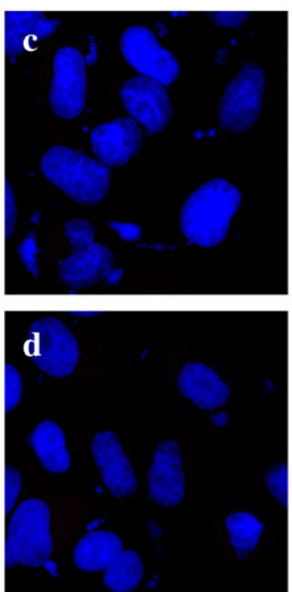

C)
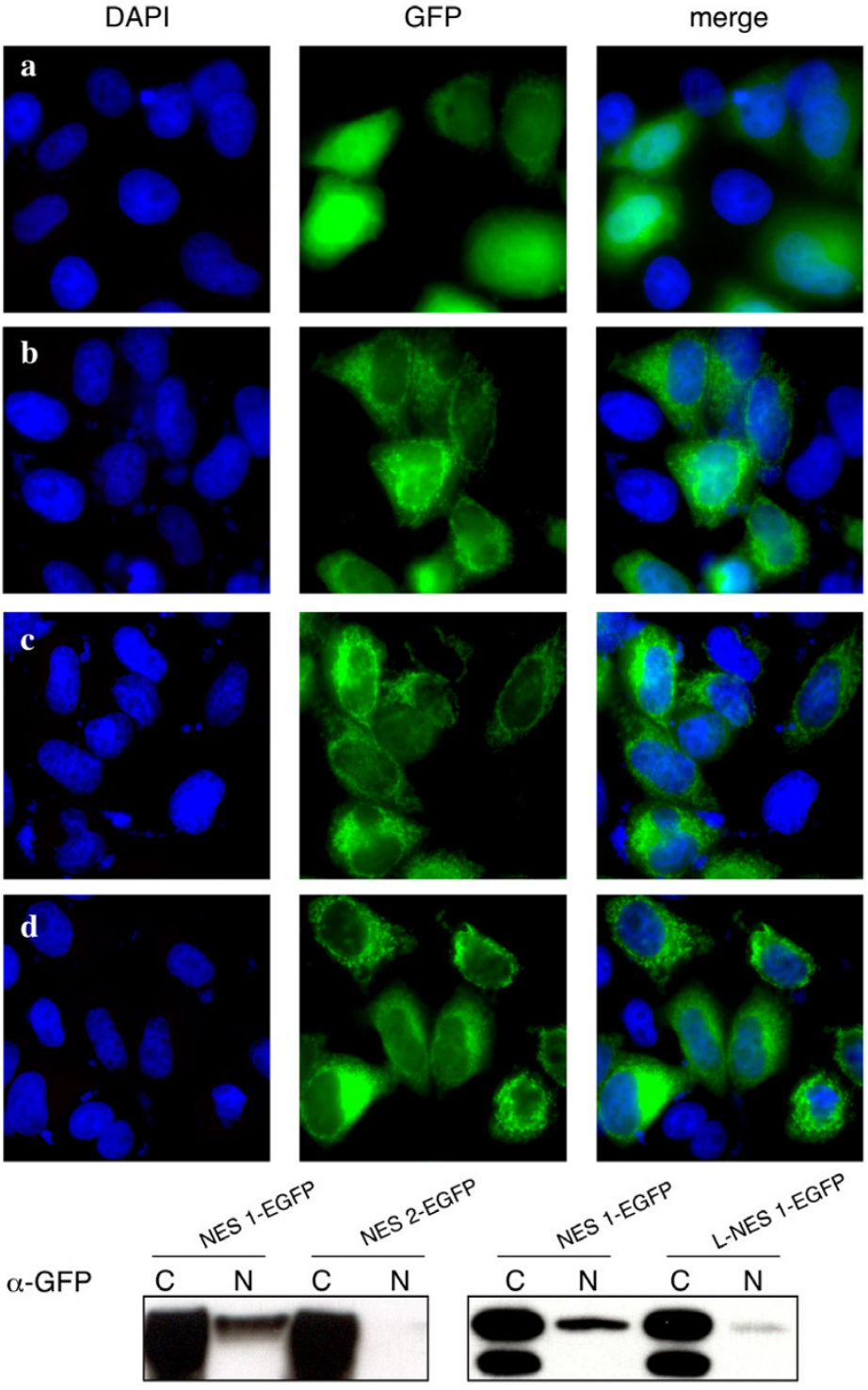

Figure 4

The presence of leucine 188 restores NESI function within the context of a NESI-EGFP protein. (A): Western-blot analysis of the different EGFP and NES-EGFP proteins. 293T cell lysates $(70 \mu \mathrm{g})$ were subjected to electrophoresis on a $10 \%$ TG gel and probed with GFP or $\beta$-tubulin antibodies. (B): HeLa cells were transiently transfected with the different NES-EGFP plasmids. Eighteen hours post transfection, transfected cells were washed, fixed, mounted with DAPI-containing medium and visualized with a Zeiss Axioplan 2 imaging microscope X40 using a Zeiss Axiocam HRc (color) camera and the Zeiss Apotome software. Images of cells that are representative of the entire population are shown. (C): 293T nuclear and cytoplasmic cell fractions were subjected to electrophoresis on a $10 \%$ TG gel and probed with a GFP antibody. The western-blot results are representative of four independent experiments. 


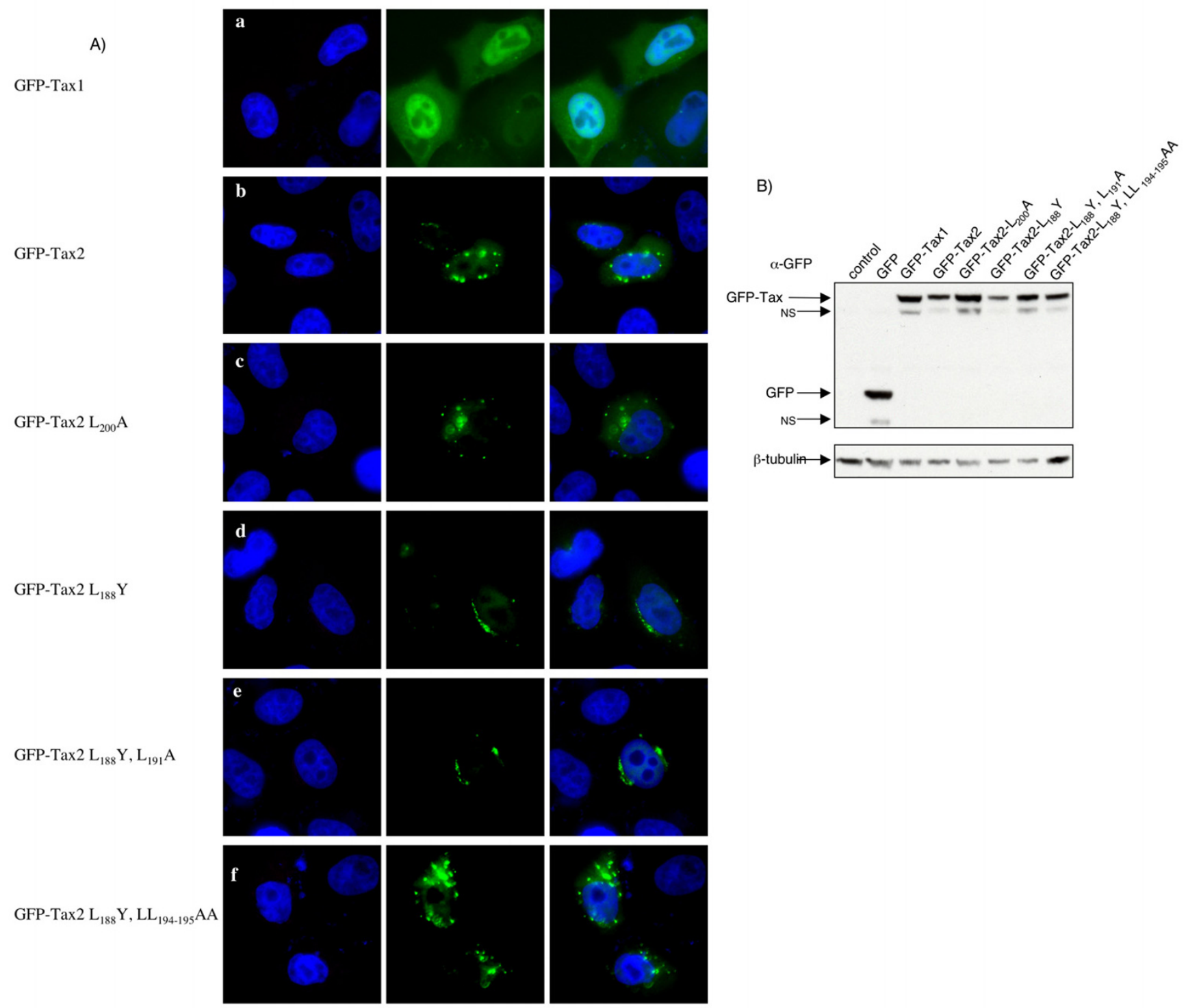

Figure 5

Within the context of a full length Tax2 protein, the presence of a functional NES2 domain is dispensable for the protein localization. (A): HeLa cells were transiently transfected with the GFP-TaxI, GFP-Tax2 and the different GFP-Tax2 mutants plasmids. Eighteen hours post transfection, the cells were washed, fixed, mounted with DAPI-containing medium and visualized with a Zeiss Axioplan 2 imaging microscope X40 using a Zeiss Axiocam HRc (color) camera and the Zeiss Apotome software. Images of cells that are representative of the entire population are shown. (B): Western-blot analysis of GFP and GFP-NES proteins. $293 \mathrm{~T}$ cell lysates $(70 \mu \mathrm{g})$ were subjected to electrophoresis on a $10 \%$ TG gel and probed with GFP or $\beta$-tubulin antibodies.

well as by our in vitro data. However, Tax2 does not need its NES signal to relocate to the cytoplasm. Rather, it seems to employ a different, hitherto uncharacterized translocation system, as we have previously suggested [16]. The implications of this paradox are that, even though a fully functional nuclear export signal is embedded in the Tax 2 sequence, it is not actually necessary for the protein translocation under the conditions tested here. However, its functional conservation suggests that it might have a biological impact on the protein functions. Future in vivo studies will decipher whether the presence of the "NES" sequence in the HTLV-2 Tax protein has any role during the viral cycle. 


\section{Methods}

\section{Cell culture and drug treatment}

Hela and 293T cells were grown in Dulbecco's modified Eagle's medium supplemented with $10 \%$ fetal bovine serum and antibiotics (penicillin $100 \mathrm{U} / \mathrm{ml}$ and streptomycin at $100 \mu \mathrm{g} / \mathrm{ml}$ ). Cell lines were maintained at $37^{\circ} \mathrm{C}$ in $5 \% \mathrm{CO}_{2}$. When indicated, cells were incubated with leptomycin B (Sigma) at $40 \mathrm{nM}$ for $3 \mathrm{~h}$.

\section{GFP-NES, NES-EGFP and GFP-Tax protein construction}

The GFP-NES and NES-EGFP recombinants plasmids were obtained by cloning double stranded oligonucleotides into GFP-C3 and EGFP-N1 vectors (Clontech), using SacI/ EcoRI and XhoI/PstI restriction sites respectively. Single or combined point mutations (at amino acids 188, 191, 194, 195 and 200) were also made in GFP-Tax1 and GFP-Tax2 sequences using the quick change mutagenesis kit (Stratagene) [16]. The nucleotide sequences of all constructs were determined using the DYEnamic ET Terminator Cycle Sequencing Kit (Amersham Biosciences) on an Applied Biosystems 373A DNA sequencer. Of note, during the course of these experiments, we noticed that the amino-acid numbering that has been used in Alefantis article was incorrect [27]. The first lysine of the Tax1 NES sequence is at position 189 and not 188 as reported previously. We have therefore modified the amino acid number accordingly.

\section{Transient transfection}

For microscopic analyses, Hela cells were seeded in eightwell chamber glass slides, at a concentration of $3 \times 10^{4}$ cells/well and transfected the next day with $0,3 \mu \mathrm{g}$ of DNA using the Effectene reagent (Qiagen). For immunoblot analyses, 293T cells were seeded on 6-well plates at $6 \times$ $10^{5}$ cells/well and transfected the next day with $2 \mu \mathrm{g}$ of DNA using the Polyfect reagent (Qiagen) following the manufacturer's instructions.

\section{Immunoblot analyses}

Twenty-four hours after transfection, 293T cells were

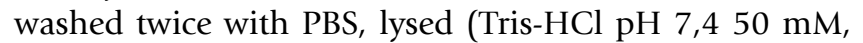
$\mathrm{NaCl} 120 \mathrm{mM}$, EDTA $5 \mathrm{mM}$, NP40 0,5\%, $\mathrm{Na}_{3} \mathrm{VO}_{4} 0,2$ $\mathrm{mM}$, DTT $1 \mathrm{mM}$, PMSF $1 \mathrm{mM}$ ) in the presence of protease inhibitors (Complete, Boehringer) and incubated on ice. Cell debris were pelleted by centrifugation. Protein concentration was determined by Bradford (Biorad). Samples were loaded into $10 \%$ Tris/Glycine gels (Invitrogen) subjected to electrophoresis at $130 \mathrm{~V}$ and transferred onto a nitrocellulose membrane (Immobilon-P, Millipore). Membranes were blocked in a 5\% PBS-milk solution, incubated with a specific anti-GFP antibody (JL-8, BD 1:1000) overnight at $4^{\circ} \mathrm{C}$. The next day, the membranes were washed and incubated with an anti-mouse horseradish peroxidase-conjugated secondary antibody (Amersham Biosciences 1:40000) and developed using the
SuperSignal West Pico Kit (Pierce). To control for the amount of protein loaded per well, membranes were stripped with the Re-blot Plus Kit (Chemicon International), and re-probed with a specific anti $\beta$-tubulin antibody (sc9104 Santa Cruz Biotechnology 1:1000).

\section{Green fluorescent protein analyses}

Twenty-four hours after transfection, the cells were washed with PBS, fixed with $4 \%$ paraformaldehyde (Sigma) and washed with PBS. Nucleic acids were stained with 4'-6'-diamine-2 phenylindole dihydrochloride (DAPI)-containing mounting medium (Vectashield, Vector). Cells were visualized with a Zeiss Axioplan 2 imaging microscope X40 using a Zeiss Axiocam HRc (color) camera and the Zeiss Apotome software. Given the fact that the localization of the GFP-fusion proteins is similar in Hela and in 293T, and because 293T cells are complex to handle in immunofluorescence experiments, we used these cells only for the western-blot analyses.

\section{Nuclear and cytoplasmic extraction}

Twenty-four hours after transfection, the cells were washed with PBS. Nuclear and cytoplasmic fractions were then isolated using the sub-cellular proteome extraction kit (Calbiochem) following the manufacturer's instructions. Samples were subjected to immunoblot analyses as described above.

\section{NetNES software analysis}

This software predicts leucine-rich nuclear export signals (NES) in eukaryotic proteins using a combination of neural networks (NN) and hidden Markov models (HMM). The prediction server calculates a combined 'NES score' from the NN and HMM scores. If the calculated 'NES score' exceeds the threshold, then that particular residue is expected to participate in a nuclear export signal. This is denoted with a "Yes" in the column "Predicted". Of note, the reason why one gets different scores for the same residues when comparing Tax 1 and Tax 2 sequences is that the score depends not only on the residue in question, but also on a number of previous residues which, in the case of E193 for example, are not identical between the two sequences.

\section{Competing interests}

The author(s) declare that they have no competing interests.

\section{Authors' contributions}

SAC, SC and LM performed the laboratory work. AG was involved in drafting the manuscript. LK participated in the interpretation of the NetNES server results and helped drafting the manuscript. RM designed, implemented and coordinated the study and wrote the manuscript. All authors have read and approved the manuscript. 


\section{Acknowledgements}

This work was funded by Institut Pasteur, by grants from l'Association de Recherche sur le Cancer (ARC \# 478I) and from ARECA to RM, fellowships from le Ministère de la Recherche to SAC, from CANAM and Pasteur Weizmann fellowships to LM and from Association Virus Cancer Prévention and La Ligue Contre le Cancer to SC. RM is supported by INSERM.

\section{References}

I. Feuer G, Green PL: Comparative biology of human T-cell lymphotropic virus type I (HTLV-I) and HTLV-2. Oncogene 2005, 24(39):5996-6004

2. Gessain A, Barin F, Vernant JC, Gout O, Maurs L, Calender A, de The $G$ : Antibodies to human T-lymphotropic virus type-I in patients with tropical spastic paraparesis. Lancet 1985, 2(8452):407-4I0.

3. Murphy EL, Fridey J, Smith JW, Engstrom J, Sacher RA, Miller K, Gibble J, Stevens J, Thomson R, Hansma D, Kaplan J, Khabbaz R, Nemo G: HTLV-associated myelopathy in a cohort of HTLV-I and HTLV-II-infected blood donors. The REDS investigators. Neurology 1997, 48(2):315-320.

4. Poiesz BJ, Ruscetti FW, Gazdar AF, Bunn PA, Minna JD, Gallo RC: Detection and isolation of type $C$ retrovirus particles from fresh and cultured lymphocytes of a patient with cutaneous T-cell lymphoma. Proc Natl Acad Sci U S A 1980, 77(12):7415-7419.

5. Grassmann R, Aboud M, Jeang KT: Molecular mechanisms of cellular transformation by HTLV-I Tax. Oncogene 2005, 24(39):5976-5985.

6. Jeang KT, Giam CZ, Majone F, Aboud M: Life, death, and tax: role of HTLV-I oncoprotein in genetic instability and cellular transformation. J Biol Chem 2004, 279(3 I):3199|-31994.

7. Mahieux R, Pise-Masison CA, Lambert PF, Nicot C, De Marchis L, Gessain A, Green P, Hall W, Brady JN: Differences in the ability of human T-cell lymphotropic virus type I (HTLV-I) and HTLV-2 tax to inhibit p53 function. I Virol 2000, 74( I 5):6866-6874.

8. Mahieux R, Pise-Masison CA, Nicot C, Green P, Hall WW, Brady JN: Inactivation of p53 by HTLV type I and HTLV type 2 Tax trans-activators. AIDS Res Hum Retroviruses 2000, 16(16): 1677-168I.

9. Ross TM, Minella AC, Fang ZY, Pettiford SM, Green PL: Mutational analysis of human T-cell leukemia virus type 2 Tax. J Virol 1997, 7 I(I I):8912-8917.

10. Ross TM, Pettiford SM, Green PL: The tax gene of human T-cell leukemia virus type 2 is essential for transformation of human T lymphocytes. J Virol 1996, 70(8):5194-5202.

I I. Semmes OJ, Majone F, Cantemir C, Turchetto L, Hjelle B, Jeang KT: HTLV-I and HTLV-II Tax: differences in induction of micronuclei in cells and transcriptional activation of viral LTRs. Virology 1996, 2 I 7(I):373-379.

12. Sieburg M, Tripp A, Ma JW, Feuer G: Human T-cell leukemia virus type I (HTLV-I) and HTLV-2 tax oncoproteins modulate cell cycle progression and apoptosis. J Virol 2004, 78(19): 10399-10409.

13. Tanaka Y, Hayashi M, Takagi S, Yoshie O: Differential transactivation of the intercellular adhesion molecule I gene promoter by TaxI and Tax 2 of human T-cell leukemia viruses. J Virol I996, 70( (12):8508-8517.

14. Tripp A, Liu Y, Sieburg M, Montalbano J, Wrzesinski S, Feuer G: Human T-cell leukemia virus type I tax oncoprotein suppression of multilineage hematopoiesis of CD34+ cells in vitro. J Virol 2003, 77(22): I2I $152-12164$.

15. Tsubata C, Higuchi M, Takahashi M, Oie M, Tanaka Y, Gejyo F, Fujii $M$ : PDZ domain-binding motif of human $T$-cell leukemia virus type I Tax oncoprotein is essential for the interleukin 2 independent growth induction of a T-cell line. Retrovirology 2005, 2:46.

16. Meertens L, Chevalier S, Weil R, Gessain A, Mahieux R: A I O-amino acid domain within human $T$-cell leukemia virus type $I$ and type 2 tax protein sequences is responsible for their divergent subcellular distribution. J Biol Chem 2004, 279(4I):43307-43320.

17. Meertens L, Pise-Masison C, Quere N, Brady J, Gessain A, Mahieux R: Utilization of the CBP but not the p300 co-activator by human T-lymphotropic virus type-2 Tax for p53 inhibition. Oncogene 2004, 23(32):5447-5458.

18. Niinuma A, Higuchi M, Takahashi M, Oie M, Tanaka Y, Gejyo F, Tanaka N, Sugamura K, Xie L, Green PL, Fujii M: Aberrant activation of the interleukin-2 autocrine loop through the nuclear factor of activated $T$ cells by nonleukemogenic human $T$-cell leukemia virus type 2 but not by leukemogenic type I virus. J Virol 2005, 79( I 8): I I925-I I934.

19. Endo K, Hirata A, Iwai K, Sakurai M, Fukushi M, Oie M, Higuchi M, Hall WW, Gejyo F, Fujii M: Human T-cell leukemia virus type 2 (HTLV-2) Tax protein transforms a rat fibroblast cell line but less efficiently than HTLV-I Tax. I Virol 2002, 76(6):2648-2653.

20. Fahrenkrog B, Koser J, Aebi U: The nuclear pore complex: a jack of all trades? Trends Biochem Sci 2004, 29(4): I75-182.

21. Xu L, Massague J: Nucleocytoplasmic shuttling of signal transducers. Nat Rev Mol Cell Biol 2004, 5(3):209-219.

22. Quensel C, Friedrich B, Sommer T, Hartmann E, Kohler M: In vivo analysis of importin alpha proteins reveals cellular proliferation inhibition and substrate specificity. Mol Cell Biol 2004, 24(23): 10246-10255.

23. la Cour T, Kiemer L, Molgaard A, Gupta R, Skriver K, Brunak S: Analysis and prediction of leucine-rich nuclear export signals. Protein Eng Des Sel 2004, $17(6): 527-536$.

24. la Cour T, Gupta R, Rapacki K, Skriver K, Poulsen FM, Brunak S: NESbase version I.0: a database of nuclear export signals. Nucleic Acids Res 2003, 3 I ( I):393-396.

25. Daelemans D, Costes SV, Lockett S, Pavlakis GN: Kinetic and molecular analysis of nuclear export factor CRMI association with its cargo in vivo. Mol Cell Biol 2005, 25(2):728-739.

26. Yashiroda $Y$, Yoshida M: Nucleo-cytoplasmic transport of proteins as a target for therapeutic drugs. Curr Med Chem 2003, I 0(9):74l-748.

27. Alefantis T, Barmak K, Harhaj EW, Grant C, Wigdahl B: Characterization of a nuclear export signal within the human $T$ cell leukemia virus type I transactivator protein Tax. J Biol Chem 2003, 278(24):218|4-21822.

28. Felber BK, Paskalis H, Kleinman-Ewing C, Wong-Staal F, Pavlakis GN: The $\mathrm{pX}$ protein of HTLV-I is a transcriptional activator of its long terminal repeats. Science 1985, 229(47| 4):675-679.

29. Goh WC, Sodroski J, Rosen C, Essex M, Haseltine WA: Subcellular localization of the product of the long open reading frame of human T-cell leukemia virus type $I$. Science 1985 , 227(469 I): I 227- I 228.

30. Kiyokawa T, Kawaguchi T, Seiki M, Yoshida M: Association of the pX gene product of human T-cell leukemia virus type-I with nucleus. Virology 1985, I47(2):462-465.

31. Slamon DJ, Press MF, Souza LM, Murdock DC, Cline MJ, Golde DW, Gasson JC, Chen IS: Studies of the putative transforming protein of the type I human T-cell leukemia virus. Science 1985 , 228(4706): I427-I430.

32. Smith MR, Greene WC: Characterization of a novel nuclear localization signal in the HTLV-I tax transactivator protein. Virology 1992, I87( I):316-320.

33. Semmes OJ, Jeang KT: Localization of human T-cell leukemia virus type I tax to subnuclear compartments that overlap with interchromatin speckles. J Virol 1996, 70(9):6347-6357.

34. Burton M, Upadhyaya CD, Maier B, Hope TJ, Semmes OJ: Human Tcell leukemia virus type I Tax shuttles between functionally discrete subcellular targets. J Virol 2000, 74(5):235 I-2364.

35. Turci M, Romanelli MG, Lorenzi P, Righi P, Bertazzoni U: Localization of HTLV-2 Tax protein is dependent upon a nuclear localization determinant in the $\mathbf{N}$-terminal region. Gene in press.

36. Fornerod M, Ohno M, Yoshida M, Mattaj IW: CRMI is an export receptor for leucine-rich nuclear export signals. Cell 1997, 90(6): | 051 - 1060.

37. Sun SC, Yamaoka S: Activation of NF-kappaB by HTLV-I and implications for cell transformation. Oncogene 2005, 24(39):5952-5964. 